\title{
MODIFIED PRONE POSITIONING AFTER SPINAL ANAESTHESIA FOR TENDON ACHILLES SURGERY; RETROSPECTIVE ANALYSIS OF 25 CASES
}

\author{
Ojiakor, S.C ${ }^{1}$, Balavenkatsubraminan, J. ${ }^{2}$ and Amit, D. ${ }^{2}$
}

1. Department of Anaesthesia \& Intensive Care, Nnamdi Azikiwe University Teaching Hospital Nnewi, Anambra State, Nigeria.

2. Department of Anaesthesiology, Ganga Hospital, Coimbatore, India.

Corresponding Author: Dr. Ojiakor Samuel Chukwudi, Department of Anaesthesia \& Intensive Care, Nnamdi Azikiwe University Teaching Hospital Nnewi, Anambra State, Nigeria. Email: drojiakorsc@yahoo.com. +2348037408192.

\section{Abstract}

This retrospective study aims to establish that modified prone positioning after spinal anaesthesia is safe and provides optimal surgical conditions for Tendon Achilles and related limb procedures.

\section{Methods}

Twenty five patients who had undergone tendon Achilles repair under spinal anaesthesia in modified prone positions at Ganga Hospital India, between January 2011 and December 2012, were enrolled into the study.

\section{Results}

Patients were reported to be comfortable throughout the procedure and there was no incidence of hypotension, bradycardia, failed block or conversion to general anaesthesia.

\section{Conclusion}

Modified prone positioning after sub-arachnoid block are comfortable for patients and provide optimal condition for the surgery.

Keywords: Modified Prone Position Spinal Anaesthesia Tendon Achilles 


\section{Introduction}

The goal of surgical positioning in anaesthesia is to ensure balance between the risk to the patient and facilitation of optimum surgical access. Proper positioning of surgical patient is critical to safe outcome and requires that all potential pressure areas protected, maintenance of adequate ventilation, and uninterrupted haemodynamic circulation with overall patient comfort. There are considerable issues associated with turning of patient shortly after induction of spinal anaesthesia which largely depends on which position was adopted. Some of these complications include, migration of the height of block to unanticipated high level, hypotension, bradycardia, failed spinal and cardiopulmonary arrest. Tendon Achilles surgery is a relatively short and uncommon procedure often performed in prone position under general anaesthesia and a few with local anaesthetic infiltration ${ }^{1}$. The desire to avoid general anaesthesia and its potential risks necessitated the adoption of 'MODIFIED PRONE POSITION' after sub-archnoid block for all the fit patient who had tendon Achilles repair.

\section{Methods}

After institutional review board approval was obtained, twenty five (25) case notes retrieved from medical records department of tendon achilles surgeries carried out in Ganga hospital between January 2011 to December 2012 were recruited into the study. From the anaesthetic chart, age, sex, volume, and type of local anaesthetic agent used as well as conduct of anaesthesia were obtained. Standard monitoring was carried out for all the patient including electrocardiogram, non-invasive blood pressure measurement and pulse oximetry. Spinal anaesthesia were performed following standard asceptic protocol with size $25 \mathrm{G}$ spinal needle in sitting position and thereafter patient was turned to modified prone position. Data collected were analyzed using SPSS window 16 software programme.

\section{Positioning}

The patient lying prone with slight tilt to the side. The two arms abducted while one resting on the arm board so that the head and neck is free and can be moved sideways. The limb to be operated is placed straight exposing the operation field while the other limb was flexed both at the hip and knee such that one side of the body is slightly raised higher than the other. See the positions below: figures 2 and 3 .
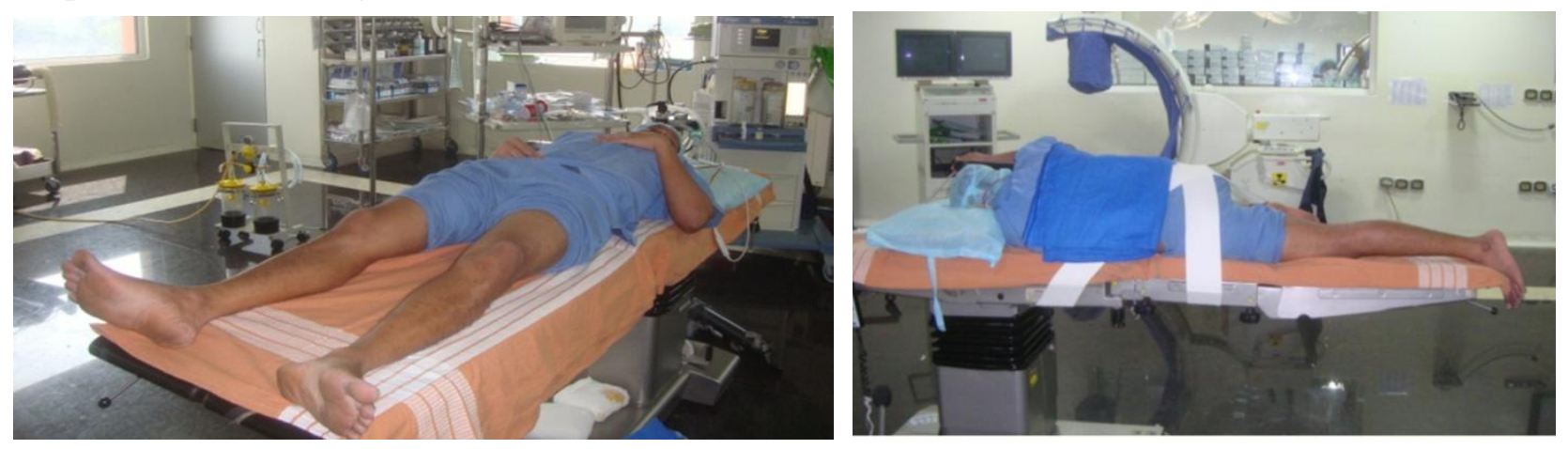


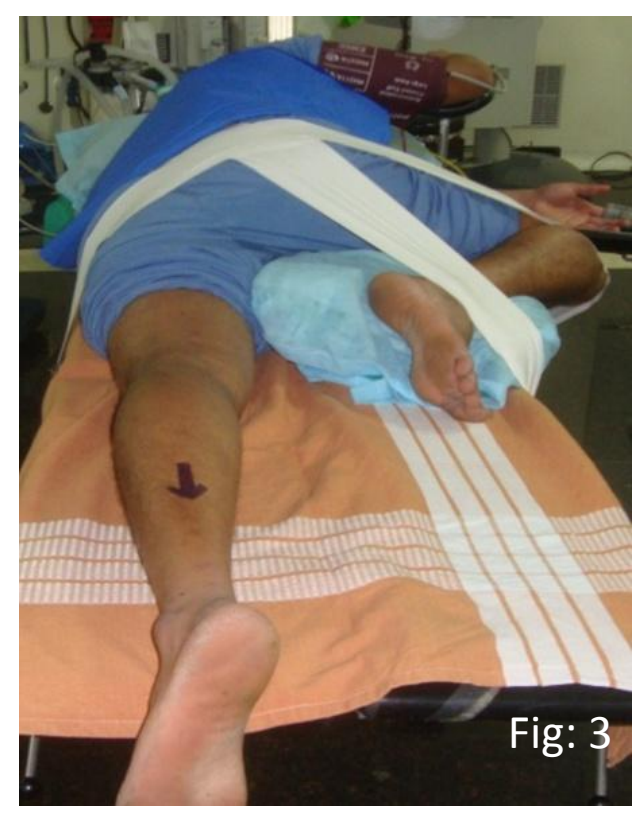

\section{Results}

Twenty five case notes were recruited into the study: fifteen $(60 \%)$ were males while 10 $(40 \%)$ were females. The minimum and the maximum ages of patients were 15 and 74 years respectively. The volume of anaesthetic agent used varied from $2.0 \mathrm{ml}-2.4 \mathrm{ml}$ of $0.5 \%$ hyperbaric bupivacaine. After the induction of anaesthesia, the systolic blood pressure (SBP) diastolic blood pressure (DBP), mean arterial pressure (MAP) and heart rate decreased significantly compared with the baseline values (P value <0.001) (figure 4). Although the change in the blood pressure and heart rate was statistically, there was no corresponding clinical significance as none of the patients developed hypotension or bradycardia. One remarkable observation in all the patient was the recovery of the haemodynamic variable about 20 minutes after block to values close to that of the baseline. This finding is consistent with traditional pattern of recovery following uncomplicated spinal anaesthesia. The entire patient maintained oxygen saturation between $98 \%$ - 100\%. No neurological sequalae reported while average length of stay in the hospital was 4 days. 


\section{Figure 4: The vitals at baseline and at various intervals after spinal block}

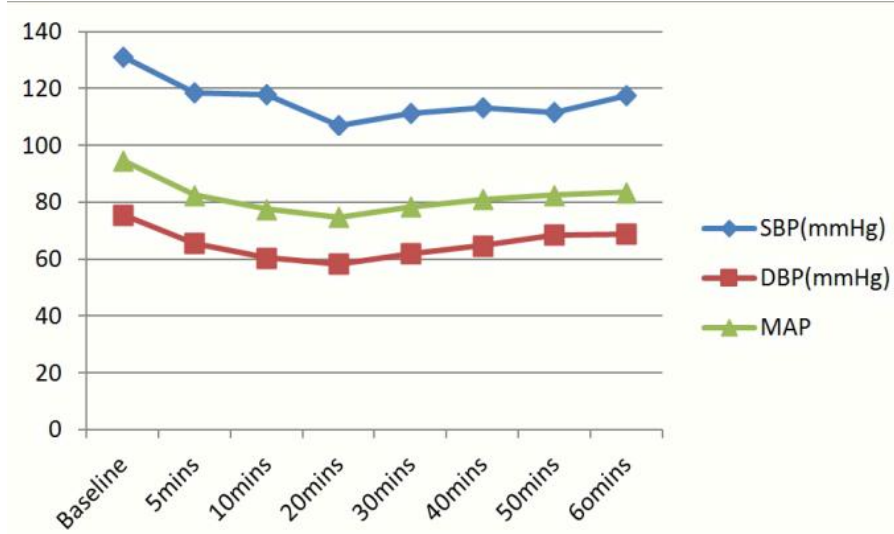

Note: SBP; systolic blood pressure, DBP; diastolic blood pressure, MAP; mean arterial pressure

\section{Discussion}

Our observation through the study shows that the patients were comfortable throughout the procedure. There was no incidence of hypotension, bradycardia or conversion to general anaesthesia. The transient drop in blood pressure noticed was consistent with changes normally associated with spinal anaesthesia in relation to the extent of sympathetic afferent nerve fibres blockade which in turn corresponds to the height of block ${ }^{4}$. Modified prone position after spinal anaesthesia provided a well anaesthesized operation field to the surgeon's admiration, reduced blood loss, excellent postoperative analgesia and reduced risk of deep vein thrombosis. Although some authors ${ }^{2,3}$ have argued the safety of patient placement in prone position after spinal anaesthesia because of possible distension of epidural veins which may lead to abnormally high block. This study did not record any untoward haemodynamic complications or augmentation of the spinal anaesthesia. We have assumed however that the thoracic lordosis which became very prominent in the position may have helped as the anatomical and physiological barrier to the cephalad migration of the local anaesthetic agent from reaching the higher thoracic nerve roots.

\section{Conclusion}

This study demonstrates that modified prone position after spinal anaesthesia is safe and provided optimal surgical condition for tendon Achilles procedures.

\section{Conflict of interest: None}




\section{References}

1. Jun A, Masakazu H, Yujisugimoti, Hirokazu I, Yoshiko T, Akira K. Spread of spinal anaesthesia in patient having perianal surgery in jack knife position: e ects of baricity of $0.5 \%$ bupivacaine and positioning during and after induction of spinal anaesthesia. $\mathrm{J}$ clin anaesth 2009; 21(10): 408-413.

2. Macquet AJ, Christensen RJ, Debenham M, Wyatt M, Panting AL. Open repair of acutely torn tendon Achilles under local anaesthetic. ANZ J Surg, 2011; 81(9): 619623.

3. Mehrabis S, Karimzadesh shirazi K. Results and complications of spinal anaesthesia inpercutaneous nephrolithotomy. Urol J 2010; 7(1): 22-25.

4. Barash PG, Gullen BF, Stoelting RK. Clinical Anaesthesia, 2006 :700-704. Lipincoth Williams \& Wilkins philadephia, USA. 